\title{
Calidad de vida y vejez masculina en México
}

\author{
Quality of life and male old age in Mexico
}

\author{
Rosa María Flores Martínez1 \\ Sagrario Garay Villegas ${ }^{2}$
}

\section{Resumen}

El objetivo de este escrito es presentar un análisis de la calidad de vida de hombres adultos mayores residentes de tres entidades mexicanas, a partir de algunas dimensiones vinculadas con los entornos físicos y sociales. Su relevancia se sustenta en dos puntos: por un lado, debido a la falta de desarrollo de esta línea de investigación (vejez), dentro del estudio de las masculinidades; por otro lado, por el progresivo proceso de envejecimiento que se presenta en México que plantea retos importantes en materia de política social con perspectiva de género.

En el artículo se plasman algunas reflexiones en torno al proceso de envejecimiento en términos demográficos, diferenciando a partir del sexo de las personas adultas mayores. Enseguida, se realizan algunas precisiones teórico-conceptuales enfocadas en el tema de las masculinidades y la calidad de vida en la vejez. Posteriormente, se desarrolla la metodología, misma que es de corte cuantitativo y transversal, en donde se retoman datos de la Encuesta sobre Condiciones de Vida de las Personas Adultas Mayores en México, 2016.

A partir de la información obtenida en la encuesta se esboza el análisis de los resultados, en el cual se evidencia que en la vejez los varones enfrentan vulnerabilidad, especialmente respecto a su entorno social, lo cual puede llegar a afectar su calidad de vida. Finalmente, el trabajo concluye con algunas reflexiones y propuestas analíticas que contemplan visibilizar las condiciones y la calidad de vida de la población adulta mayor masculina en México.

Palabras clave: calidad de vida, vejez, género, masculinidades

\section{Abstract}

The objective of this paper is to present an analysis of the quality of life of older adult men residing in three Mexican entities, based on some dimensions related to physical and social environments. Its relevance is based on two points: on the one hand, due to the lack of development of this line of research (old age), within the study of masculinities; on the other hand, due to the progressive aging process that

\footnotetext{
Recibido: 06 de marzo de 2019 Aceptado: 31 de octubre de 2019 Publicado: 20 de diciembre de 2019 ${ }^{1}$ Estudiante del programa de Doctorado, en el Posgrado de la Facultad de Trabajo Social y Desarrollo Humano de la UANL. Correo electrónico: rosamariaflores1@gmail.com

${ }^{2}$ Profesora-Investigadora de tiempo completo, en el Posgrado de la Facultad de Trabajo Social y Desarrollo Humano de la UANL. Correo electrónico: sgarayv@gmail.com
} 
occurs in Mexico that poses important social policy challenges with a gender perspective.

The article reflects some reflections on the aging process in demographic terms, differentiating from the sex of the elderly. Next, some theoretical and conceptual clarifications are made focused on the issue of masculinities and the quality of life in old age. Subsequently, the methodology is developed, which is quantitative and transversal, where data are collected from the Survey on Living Conditions of Older Adults in Mexico, 2016.

Based on the information obtained in the survey, the analysis of the results is outlined, whose information shows that in old age men face vulnerability, especially with respect to their social environment, which may affect their quality of life. Finally, the work concludes with some reflections and analytical proposals that contemplate making visible the conditions and quality of life of the elderly male population in Mexico.

Keywords: quality of life, old age, gender, masculinities

\section{Proceso de envejecimiento demográfico en México}

A nivel global el envejecimiento poblacional es un fenómeno que ha marcado las últimas décadas del siglo $\mathrm{XX}$ y las primeras del presente. De acuerdo con las Naciones Unidas (2003), este proceso demográfico sucede de manera vertiginosa, especialmente en los países en desarrollo y en algunos países con economías en transición. En términos generales, el envejecimiento de la población es un proceso heterogéneo, caracterizado precisamente por su diversidad, que acontece de forma distinta según las características y condiciones de cada región.

En México, Partida (2005) señala que el proceso de envejecimiento demográfico está sucediendo a un ritmo acelerado, en comparación con otras regiones más desarrolladas. Al respecto, el Instituto Nacional de Estadística y Geografía (INEGI, 2015) señala, según datos de la Encuesta Intercensal 2015, que del total de la población en el territorio mexicano $10.4 \%$ son personas de 60 años o más, de las cuales $53.7 \%$ son mujeres y $46.3 \%$ son varones, de estos últimos la mayor concentración se presenta en el grupo de menor edad (31.3\%), disminuyendo la proporción conforme se incrementa la edad, sin embargo, en el grupo de 75 años y más se presenta un ligero incremento (27.0\%) (INEGI, 2015).

De acuerdo con estimaciones del Consejo Nacional de Población (CONAPO, 2018), en el país, para el año 2019 la esperanza de vida en promedio para la población es de 75.1 años; cabe señalar que la esperanza de vida es más elevada en el caso de las mujeres (77.9 años), en comparación con los hombres (72.2 años), lo cual se prevé sea una tendencia en las próximas décadas. Pese a que la esperanza de vida es más elevada para las mujeres, ellas viven esos años adicionales con mayor discapacidad y peores condiciones de salud que los varones (Gutiérrez, Agudelo, Giraldo y Medina, 2016).

El envejecimiento poblacional de hombres y mujeres, así como el aumento de la esperanza de vida desde el nacimiento representan logros importantes, no obstante, al mismo tiempo traen consigo importantes desafíos, principalmente en términos políticos, económicos y sociales, tanto para las personas adultas mayores como para 
las familias, la sociedad y el Estado, no por el incremento en términos absolutos y relativos de este grupo etario, sino por la capacidad para abordar y hacer frente a los desafíos que conlleva este proceso, que forma parte de la realidad cotidiana que se vive en de diversos países, incluido México.

\section{Vejez y masculinidades}

Las relaciones de género en el curso de la vida no son inamovibles, al contrario, en éstas se producen diversas transiciones que, en vinculación con las normas basadas en la edad y los cambios fisiológicos, inciden en el modo de interpretar las funciones atribuidas a los géneros (Ginn y Arber, 1996). De esta forma, tal y como señalan las autoras, el género y el envejecimiento están estrechamente conectados con la vida social, de modo que cada uno sólo puede entenderse en relación con el otro.

De manera que, género y envejecimiento son dos dimensiones concatenadas tanto a nivel estructural, como social e individual, permeadas por continuidades y discontinuidades que construyen y deconstruyen esta relación a lo largo del tiempo. Así, durante el curso de vida las personas están constantemente influidas por el contexto social, económico, cultural y político en el que viven, de igual manera, por las construcciones sociales en torno al género y a la edad, así como por las relaciones que se producen a partir de éstos.

La construcción social de la vejez no se produce en un vacío social sino dentro de un contexto histórico y social (Kehl y Fernández, 2001), por lo que la imagen social y el significado de ser viejo atiende a una determinada realidad social, en donde el lenguaje constituye el principal sistema de símbolos a través del cual las personas aprenden, producen e intercambian significados; por ejemplo, mientras que la juventud es asociada a significados positivos como la belleza, la energía y la vitalidad; en tanto en la vejez adquiere connotaciones negativas, como la inactividad, la fealdad y la enfermedad. Sin embargo, cabe enfatizar que estas polaridades simbólicas nunca son definitivas y estáticas, sino que se transforman históricamente (Nederveen, 1992; Featherstone y Wernick, 1995 citados en Kehl y Fernández, 2001).

El proceso de envejecer se relaciona con las transiciones que se presentan en el curso vital, no obstante, tanto el momento como la sucesión de tales transiciones son diferentes según se trate de mujeres y hombres, por lo que la vejez social está marcada por el género; así las normas culturales para la posición en espacios reproductivos y productivos, cambia de un género a otro.(Ginn y Arber, 1996).

Cuando se habla de género necesariamente se tiene que señalar que es una categoría dinámica construida socialmente, que tiene como fundamento las diferencias sexuales biológicas, así como el contexto y el ambiente social que determinan las expresiones de los comportamientos y los roles sociales atribuidos a hombres y mujeres. (Careaga, 1996 citado en Hardy y Jiménez, 2001).

Al analizar la condición varones se han identificado diversas perspectivas que abordan el tema de la masculinidad, en este sentido existen posiciones conservadoras que conciben la masculinidad como una determinación del patriarcado en donde los varones son asociados a nociones de liderazgo, poder y autoridad, mientras que las mujeres a posiciones de subordinación y esto se concibe como un continuo en la organización social; no obstante, este posicionamiento ha 
sido fuertemente criticado y cuestionado pues se considera algo universal e inamovible (Menjívar, 2010).

Por otro lado, existen posturas críticas que entienden la masculinidad no como algo estático sino como una construcción social en constante transformación y cambio en el marco de relaciones sociales conflictivas (Menjívar, 2010). De tal manera que no se podría hablar sólo de un tipo singular de masculinidad, sino de múltiples masculinidades cuyas identidades se construyen, deconstruyen y reconstruyen frente a los cambios sociales de tipo económico, político, cultural y familiar.

De acuerdo con Hardy y Jiménez (2001), la masculinidad es una construcción social, que al igual que la feminidad, comienza a edificarse incluso antes del nacimiento y continúa hasta la muerte. De esta forma, la distribución de poder, privilegios y bienestar de las personas en una la sociedad varía tanto entre los géneros como dentro de éstos, lo cual se traduce en las distintas posiciones que hombres y mujeres tienen en la jerarquía social y en las condiciones de vida manifestadas en cada etapa transicional.

En la vejez surgen nuevas masculinidades, la identidad de los varones se encuentra expuesta a una discontinuidad en los roles tradicionales fuertemente vinculados con nociones de poder, fortaleza, productividad y virilidad. En otras palabras, en la vejez los hombres enfrentan un dejar de ser, viven un proceso en el que sufren una devaluación social, en donde cada vez se le toma menos en cuenta (Ramos, 2014). Además, esta etapa coincide con el proceso de la llamada jubilación, lo cual repercute en la disminución de ingresos y en la pérdida del estatus de proveedor principal.

\section{Calidad de vida en la vejez}

El tema de la vejez remite a su abordaje no sólo en términos físicos, sino también sociales y culturales. De acuerdo con Fericgla (2002), el concepto de vejez es una construcción sociocultural, en la que intervienen diversos elementos relacionados con la salud, la situación económica, la situación familiar, la capacidad de automantenimiento y la funcionalidad.

En este sentido, es preciso señalar que no todas las personas viven la vejez de la misma forma; el funcionamiento y la calidad de vida que se presentan durante esta etapa, se encuentran vinculados con las omisiones y acciones que cada una de las personas ha realizado durante el transcurso de los años (González-Celis, 2010).

Para Fernández- Ballesteros (1997), la calidad de vida en la vejez es un tema complejo y multifactorial, que comprende distintas dimensiones -objetivas y subjetivas- igualmente se encuentra marcada por el género y la edad, así como el estrato socioeconómico, el nivel de estudios, el estado de salud, la funcionalidad y las políticas de bienestar implementadas por el Estado.

Una de las dimensiones que influye en la calidad de vida de la población adulta mayor, es contar con entornos físicos y sociales emancipadores, propicios y favorables, que den respuesta a las oportunidades y retos que implica el envejecimiento poblacional y procuren condiciones de bienestar para las personas adultas mayores (Naciones Unidas, 2003).

Dentro de los entornos sociales, "las redes sociales de apoyo son estrategias que utilizan las personas para obtener o mantener ciertos beneficios en su calidad de vida" (Montes de Oca, 2004:27). Éstas se han encargado de dar respuesta a las 
necesidades de las personas adultas mayores, desde los apoyos más básicos, hasta brindar ayuda de tipo económico e incluso emocional (CELADE-CEPAL, 2006). Cabe señalar que hoy en día existe un amplio segmento de la población que no cuenta con acceso a mecanismos institucionales para satisfacer sus necesidades básicas, por lo que depende, en gran medida, de la familia y de las redes sociales de apoyo, para poder cubrir sus requerimientos (Guzmán, Huenchuan y Montes de Oca, 2003).

Por lo que respecta a los entornos físicos, uno de los aspectos prioritarios es la vivienda; este espacio constituye un elemento clave que incide en la calidad de vida de las personas adultas mayores. Además de ser un factor de apoyo y seguridad económica en la vejez, puede ser un activo que genere el intercambio de apoyo con otros miembros de la familia, especialmente con los hijos (Garay, Montes de Oca y Hebrero, 2015). Según el CELADE-CEPAL (2006:145), "el acceso a una vivienda adecuada constituye una exigencia imprescindible para una plena satisfacción de las necesidades humanas".

En general, contar con un entorno favorable (físico y social) es uno de los aspectos prioritarios que se vinculan directamente con la calidad de vida de la población que envejece. No obstante, en la actualidad existen importantes brechas y rezagos que afectan de manera diferenciada a hombres y a mujeres. Particularmente este escrito se centra en la calidad de vida de los varones envejecidos, quienes a llegar a la vejez se ven expuestos a experimentar nuevos roles y condiciones de vida.

\section{Método}

La presente investigación analiza datos de la Encuesta sobre Condiciones de Vida de las Personas Adultas Mayores en México, 2016. Dicha investigación es de corte cuantitativo, con un diseño transversal, no experimental y ex post facto. Con una muestra constituida por 1152 casos, calculada en función de dos estratos: urbano y rural. Las entidades federativas de levantamiento de la información fueron las siguientes: Nuevo León, Jalisco y la Ciudad de México.

El instrumento está integrado por trece apartados, los cuales son descritos a continuación: 1) datos de identificación; 2) datos de los miembros del hogar; 3) datos de la persona entrevistada (sólo personas de 60 años o más); 4) datos generales sobre condiciones de la vivienda; 5) redes de apoyo (recepción de apoyos familiares y no familiares, otorgamiento de apoyos familiares y no familiares); 6) actividades y funcionalidad; 7) violencia y maltrato; 8) participación social en la vejez; 9) imagen social de la vejez; 10) uso y acceso a los espacios públicos; 11) condición y autopercepción de la salud; 12) bienestar subjetivo; 13) arreglos residenciales. De acuerdo con el interés central del estudio, del instrumento señalado anteriormente se analizan de forma principal las siguientes dimensiones: datos de la persona entrevistada, condiciones de la vivienda y redes de apoyo social.

Para fines del presente estudio, de la muestra general $(\mathrm{n}=1152)$ se seleccionó únicamente a las personas adultas mayores varones, dado que se pretende 
profundizar, particularmente en la calidad de vida de los hombres adultos mayores, a partir de los entornos físicos y sociales. Así, finalmente la muestra total de este análisis quedó conformada por $n=640$ casos, mismos que serán considerados como la totalidad de la población sujeto de estudio. Para el procesamiento de los datos se utilizó el programa Statistical Packagefor Social Science (SPSS) versión 22.0.

\section{Análisis de los resultados}

La calidad de vida de los hombres adultos mayores está relacionada con diversos aspectos, los entornos propicios y favorables son parte esencial de estos elementos. Por tanto, en este documento se asume la relevancia de analizar las condiciones de la calidad de vida en la vejez masculina a partir del entorno social y físico, particularmente ahondando en las redes de apoyo social y las condiciones de la vivienda en la que residen las personas entrevistadas.

De acuerdo con los datos del estudio, la edad de los hombres adultos mayores entrevistados oscila en un rango de entre 60 y 95 años, con una media de 69.3 años. Scott y Wenger (1996) sostienen que la diferencia entre la esperanza de vida de hombres y mujeres, influye en el estado civil de las personas adultas mayores; la menor esperanza de vida en los varones, aunado con su tendencia a casarse con mujeres menores que ellos y el hecho de que se vuelvan a casar (tras presentarse la viudez), incide en que exista mayor probabilidad de que sigan casados en la vejez, en tanto, en el caso de las mujeres es más factible que enviuden y permanezcan así hasta su muerte.

Respecto a su estado civil, $58.8 \%$ de la población se encuentran casados o unidos, en tanto $26.5 \%$ están viudos. De lo anterior, cabe destacar un aspecto pocas veces toma relevancia dentro de los estudios de vejez y masculinidad, el estado civil. En este estudio, más de la mitad de la población se encuentra casado o unido, lo cual quiere decir que no vive su vejez en soledad. Según Askham (1996), el matrimonio es una de las relaciones básicas de las personas adultas mayores (especialmente posterior a la jubilación), de forma particular para los hombres.

En relación con el nivel de escolaridad de los varones entrevistados, en el estudio se encontró que la mayoría (28.4\%) cuenta con primaria completa, mientras que sólo $3.0 \%$ refirió que tiene estudios profesionales y $12.5 \%$ no cursó ningún grado escolar. Del Popolo (2001:48) señala que "el nivel educativo de las personas influye sobre su calidad de vida”. Así, el hecho de encontrar bajos niveles de escolaridad en los varones entrevistados es un indicador de sus condiciones de vida, pues es un factor de desigualdad social. 
Ahora bien, para comenzar a abordar el tema de los entornos sociales y, en particular, las redes de apoyo social, en la investigación se preguntó a los entrevistados: en el último año ¿ha recibido algún tipo de apoyo por parte de su familia? 66.4\% manifestó que sí lo ha recibido, oscilando entre 1 y 8 el número de familiares que brindan el apoyo, aunque cabe decir que es más frecuente que sea sólo una persona quien provea el apoyo (30.6\%), evidenciando que la red familiar tiende a ser reducida. Para conocer la proximidad física de los familiares que apoyan se preguntó ¿en dónde viven los familiares que dan apoyo y que no viven con usted? La mayoría contestó que viven en otra colonia, pero en el mismo municipio (20.9\%), mostrando así, que cuentan con una red de apoyo relativamente cercana a su vivienda.

Respecto al parentesco del familiar que brinda el apoyo se observa que en $82.4 \%$ de los casos son los hijos/as quienes lo proveen, de los cuales $47.3 \%$ varones y $35.1 \%$ mujeres. Pese a que en términos porcentuales se advierte que son los hijos varones quienes brindan apoyo a sus padres adultos mayores en mayor medida, al hacer un análisis a mayor profundidad se reveló que los hijos brindan apoyo de tipo económico e instrumental, pero este apoyo es más esporádico ya que la frecuencia es de una vez al mes o entre cada dos o tres meses en la mayor parte de los casos; en cambio, las hijas brindan apoyos a través de cuidados, quehaceres de la casa o en comida y despensa, los cuales suelen ser más frecuentes (diarios, cada tercer día o cada semana).

Al distinguir a partir del género, se ha encontrado evidencia que muestra que las mujeres en la vejez mantienen redes de apoyo más extensas, basadas no sólo en la familia, sino también en amigas y vecinas; mientras que los hombres suelen estar conformadas principalmente por sus hijos e hijas (cuando los hay) y sus vecinos (Corin, 1982 citado en Scott y Wenger, 1996). Lo anterior, concuerda con lo encontrado en presente investigación, tanto las hijas como los hijos son los principales proveedores de apoyo.

Scott y Wenger (1996) refieren que conforme las personas envejecen, sus redes de apoyo se transforman, a su vez, estos cambios se encuentran marcados por el género; en el caso de los hombres, el matrimonio les resulta ser más beneficioso, pues gozan de compañía y bienestar a partir de su cónyuge, además dada la menor esperanza de vida de los varones, es más probable que durante su vejez le sobrevivan familiares cercanos, es decir, enfrenten la vejez con menor soledad, en comparación con las mujeres. Para Ramos (2014), las relaciones familiares que los varones adultos mayores establecen, con otros miembros del hogar, son vitales, ya que éstas contribuyen en el bienestar y calidad de vida de esta población, o bien, cuando no se cuenta con ello, en su deterioro. 
Durante la vejez las redes de apoyo familiar juegan un papel crucial, para hombres y mujeres, pues es en esta etapa cuando comienzan a presentarse diversas situaciones vinculadas con la aparición de enfermedades, dependencia, fragilidad y discapacidad, por lo que, las personas en la gran parte de los casos comienzan a requerir ayuda, no sólo en actividades de la vida diaria, sino también apoyo económico e incluso afectivo. Esto incide en la reconfiguración y el cambio de significado de la masculinidad en la vejez.

Por lo que respecta a las redes de apoyo fuera del núcleo familiar, se cuestionó a los entrevistados: en el último año ¿ha recibido apoyo de personas no familiares? Sólo 5.6\% contestó que sí ha recibido apoyo por parte de personas no familiares, en su mayoría han sido los vecinos quienes brindan el apoyo. Aunque en el estudio no resultó ser una proporción elevada, estas redes representan un grupo de apoyo complementario e importante que, de acuerdo con lo encontrado en el estudio, apoyan a los hombres adultos mayores, principalmente proporcionado comida o despensa.

En términos generales, se advierte que, para los hombres adultos mayores, las redes de apoyo social desempeñan un papel importante en la etapa de la vejez, especialmente las de carácter familiar, pues proveen apoyos de distintos tipos. En este sentido, Montes de Oca y Macedo (2013:175) manifiestan que "las redes sociales conforman mecanismos que procuran aminorar los efectos negativos de contextos en desventaja, que afectan de manera diferencial a grupos de la población”. De igual manera, inciden en mejorar la calidad de vida de la población; por lo que, contar con redes de apoyo social durante la vejez, que procuren apoyo (tanto formal como informal), favorece el bienestar y la calidad de vida de los hombres adultos mayores, no sólo en términos de salud, sino también afectivos y emocionales.

Ahora bien, por lo que corresponde al entorno físico de los hombres adultos mayores, se encontró empírica que refleja parte de las condiciones físicas y materiales de la vivienda de los entrevistados, que es el espacio en el que desarrollan su vida cotidiana. El análisis se realiza diferenciando entre la zona de residencia urbana y la zona rural, a partir de esta distinción, se describen las características de la vivienda, así como el acceso a servicios públicos y el equipamiento con el que cuentan.

Uno de los principales aspectos relacionados con la dimensión de la calidad habitacional de las personas mayores, se relaciona con las condiciones materiales de las viviendas (CELADE-CEPAL, 2006). Para los hombres adultos mayores contar con una vivienda propia genera mayor bienestar, pues tienen un patrimonio que al menos les asegura un espacio para vivir. 
En la investigación se encontró que los varones entrevistados generalmente son los propietarios de la vivienda que habitan, $82.9 \%$ presentan esta condición en la zona urbana y 76.4\% en la zona rural. No obstante, algunos autores (Vázquez-Honorato y Salazar-Martínez, 2010) señalan que, aunque existe cierta generalidad en el hecho de que los adultos mayores sean propietarios de una vivienda, eso no es garantía de que este espacio cuente con las condiciones de habitabilidad, seguridad y accesibilidad que se requieren para favorecer su calidad de vida.

Por lo anterior no sólo interesa conocer el estatus de propiedad, sino las condiciones materiales de la vivienda, encontrando que en la zona urbana se presentan mejores condiciones materiales, en comparación con la zona rural. En la primera, las paredes se encuentran construidas en más del $90.0 \%$ de los casos por ladrillo, block o tabique; al igual, el techo está hecho de losa de concreto en la mayoría de las viviendas; en cuanto al material del piso, si bien 52.6\% de la población tiene piso de cemento o firme, $45.2 \%$ lo tiene de madera, mosaico $\mathrm{u}$ otros recubrimientos.

En tanto, en la zona rural, aunque las viviendas están construidas con ladrillo o block (79.5\%), se observa que aún se encuentran casas construidas de adobe, con techos de lámina metálica o de cartón (15.7\%) y, en su mayoría, con pisos de cemento o firme 65.5\%). En estas zonas, el entorno físico puede ser más hostil, dado que existen mayores condiciones de pobreza y menos recursos.

Las condiciones materiales de las viviendas en los dos contextos descritos muestran diferencias en las condiciones del entorno físico de los hombres adultos mayores rurales y los urbanos. Esto da pauta a señalar que existen condiciones físicas heterogéneas, que pueden repercutir en facilitar o limitar según sea el caso la movilidad, las relaciones y el bienestar de los varones entrevistados.

En un estudio realizado sobre las condiciones de las viviendas de la población adulta mayor que habita en algunos países de América Latina, se encontró aún persisten rezagos que afectan la calidad de vida de las personas envejecidas; por ejemplo, en países como Ecuador y México existe una proporción importante de viviendas que cuentan con piso de tierra, 9.5\% y 12.3\% respectivamente, dicho porcentaje es superior al presentado en otros países (Garay, Montes de Oca y Hebrero, 2015).

Por lo que respecta a la disposición de servicios, en el estudio se observó lo siguiente: en la zona urbana, los entrevistados manifestaron que disponen del servicio de agua dentro de su vivienda en $84.0 \%$ de los casos, respecto al acceso del drenaje conectado a la red pública, éste se encuentra cubierto prácticamente en la mayoría de los casos, al igual que el servicio de electricidad y la disponibilidad de gas el interior de la vivienda.

En la zona rural, se muestra que el acceso al servicio de agua dentro de la vivienda se presenta en $74.5 \%$ de los casos, esta proporción es menor en comparación con la 
zona urbana, aquí cabe agregar que $18.6 \%$ señaló que, aunque tienen acceso la conexión, no está en el interior de la vivienda, por lo que tienen que trasladarla desde fuera; en relación con el drenaje, si bien la mayor parte de la población cuenta con este servicio, $5.0 \%$ no tiene drenaje y $2.7 \%$ utiliza fosa séptica; finalmente, la disposición de electricidad y gas para cocinar, pese a que la mayoría cuenta con ello, la proporción es menor en las comunidades rurales que en las localidades urbanas.

A partir de los datos referidos, se advierte que los hombres adultos mayores entrevistados en la investigación habitan en viviendas que presentan rezagos, especialmente en el caso del acceso al agua potable dentro de las viviendas, lo cual es más notorio en las zonas rurales. Al momento de realizar trabajo de campo, las personas expresaron que el agua no llega con suficiente presión y no alcanza a llegar hasta el interior de la vivienda, además es común que los pozos de agua presenten fallas y las autoridades no realizan acciones para resolver el problema, por lo que en ocasiones duran días sin tener agua.

En resumen, a partir de los resultados encontrados en este estudio se advierte que los varones entrevistados en este estudio presentan condiciones de vida polarizadas. Aunque la transición hacia la vejez podría repercutir en condiciones de vida desfavorables por el hecho de "dejar de ser" como señala Ramos (2014), la presente investigación muestra que los varones son acreedores de apoyo familiar, lo cual favorece su calidad de vida y bienestar, ya que reciben apoyo de diversa índole, sin embargo, también podría generar una autopercepción negativa por reconocerse como acreedores de apoyo.

Por lo que respecta al entorno físico, la propiedad de la vivienda es un indicador que genera seguridad para los varones en esta etapa de la vida, pues representa un medio a través del cual puede continuar manteniendo cierto poder y control sobre este bien y sobre los apoyos familiares, en caso de necesitarlos. Por lo que respecto a las condiciones materiales de la vivienda y el acceso a los servicios básicos es donde se encontraron mayores diferencias, esto pone al descubierto que no todos los varones envejecidos gozan de condiciones favorables, por lo que habría que considerar otras variables como la clase social, la etnia y como en este caso, el contexto de residencia.

\section{Reflexiones finales}

En la vejez se presenta una reconfiguración de las identidades masculinas, los varones asumen nuevos roles y condiciones de vida, en algunos casos manteniendo ciertas ventajas sociales y económicas, pero en otros enfrentando crisis identitaria, falta de apoyo y disminución de ingresos. Continuar pensando a los varones desde las nociones asociadas a la masculinidad hegemónica-conservadora invisibiliza a las 
masculinidades divergentes y a las masculinidades vulneradas por los mandatos patriarcales.

En ese sentido, uno de los propósitos de este escrito fue abordar la calidad de vida de los hombres adultos mayores a partir de los entornos físicos y sociales y, al mismo tiempo, plantear que existen otras masculinidades, las de los viejos. De tal manera que, este escrito plantea que la calidad de vida de los hombres adultos mayores debe considerarse como un aspecto prioritario de intervención social por parte del Estado, al igual que la calidad de vida de las mujeres, reconociendo que existen diferencias, pero pugnando por erradicar las brechas y desigualdades entre los géneros y dentro de éstos.

En general contar con redes de apoyo social en la vejez favorece la calidad de vida de la población, ya que procuran bienestar en las personas adultas mayores, al mismo tiempo que contribuye en la creación en vínculos. Dada su importancia, hay que visibilizar que no todos los varones cuentan con redes de apoyo sólidas, situaciones como la soledad, la pobreza, la dependencia o las enfermedades crónicas, puede llegar a incidir en el desvanecimiento o reducción de las redes y, aunque estos aspectos no fueron planteados en este análisis, se considera que son dimensiones que hay que continuar explorando a mayor profundidad, desde la perspectiva de género y desde las masculinidades críticas, en posteriores investigaciones.

Con respecto al entorno físico, la vivienda es un espacio vital en el que los hombres adultos mayores desarrollan su vida cotidiana, especialmente a partir del proceso de la jubilación o retiro de la actividad productiva. En el estudio se observó que existen diferencias entre las condiciones de las viviendas de la zona urbana y las de la zona rural, en la primera, se presentan condiciones más adecuadas, particularmente en el material de las viviendas y en el acceso a servicios; mientras que en las comunidades rurales enfrentan mayores rezagos. Sin embargo, consideramos que la calidad de vida respecto al entorno físico no sólo tiene que ver con las condiciones objetivas y materiales de la vivienda, sino también con aspectos subjetivos, es decir, sobre la valoración que las personas hagan sobre su entorno, en ese sentido, exhortamos a que se continúe indagando esta línea de investigación desde miradas cualitativas.

\section{Referencias bibliográficas}

Askham, J. (1996). Vida matrimonial de las personas mayores.En S. Arber y J. Ginn: Relación entre género y envejecimiento, 127-140. Madrid: Narcea.

CELADE-CEPAL (2006). Manual sobre indicadores de calid de vida ne la vejez. Santiago de Chile: Naciones Unidas. 
CONAPO. (2018) Proyecciones de la Población de México y de las Entidades Federativas, 2016-2050. https://datos.gob.mx/busca/dataset/proyecciones-de-la-poblacion-demexico-y-de-las-entidades-federativas-2016-2050 (último acceso: 4 de Enero de 2019).

Del Popolo, F. (2001). Características sociodemográficas y socioeconómicas de las personas de edad en América Latina. Santiago de Chile: Serie Población y Desarrollo. Comisión Económica para América Latina y el Caribe/Centro Latinoamericano y Caribeño de Demografía.

Fericgla, J. (2002). Envejecer. Una antropologia de la ancianidad. Barcelona : Herder.

Fernández-Ballesteros, R. (1997). Calidad de vida en la vejez: condiciones diferenciales. Anuario de Psicología, 1997: 89-104.

Garay, S; Montes de Oca, V. y Herbrero,M. (2015). Los entornos y el envejecimiento en Iberoamérica: análisis a partir de las condiciones de vivienda. Notas de Población, 109-125.

Ginn, J. y Arber, S. (1996) «Mera conexión. Relaciones de género y envejecimiento». En S. Arber y J. Ginn: Relación entre de Género y Envejecimiento, 17 34. Madrid: Narcea.

González-Celis, A. L. (2010). Calidad de vida en el adulto mayor. En L. M. Gutiérrez y H. Gutierrez: Envejecimiento humano: una visión transdisciplinaria, 365378. México, D.F: Instituto Nacional de Geriatría.

Gutiérrez, L.M; Agudelo, M; Giraldo, L. y Medina, R. (2016). Hechos y desafios para un envejecimiento saludable en México. México: Instituto Nacional de Geriatría.

Guzmán, M; Huenchuan, S. y Montes de Oca, V (2003). Redes de apoyo social de las personas mayores: marco conceptual. Notas de Población, 35-70.

Hardy, E. y Jiménez, A.L (2001). Masculinidad y género.Rev. Cubana Salud Pública, 77-88.

INEGI (2015). Encuesta 2015. http://www.inegi.org.mx/est/contenidos/Proyectos/encuestas/hogares/especiales/ ei2015/ (último acceso: 7 de Octubre de 2016).

Kehl, S. y Fernández, J.M. (2001). La construcción social de la vejez. Cuadernos de Trabajo Social. 14: 125-161. 
Menjívar, M. (2010). La masculinidad a debate. Costa Rica: FLACSO.

Montes de Oca, V (2001). Redes comunitarias, género y envejecimiento. México. México: Colección Cuadernos de Investigación, Instituto de Investigaciones Sociales-UNAM

Montes de Oca, V y Macedo, L. (2013). Redes sociales como determinantes de la salud .Montes de Oca, V. y Macedo, L: Redes sociales como determina http://www.inger.gob.mx/bibliotecageriatria/acervo/pdf/envejecimiento_y_salud_ 11.pdf (último acceso: 30 de Octubre de 2016).

Naciones Unidas. Declaración Politica y Plan de Acción Internacional de Madrid sobre el Envejecimiento. Nueva York: Naciones Unidas, 2003.

Partida, V. (2005). La transición demográfica y el proceso de envejecimiento en México,Papeles de población, 9-27.

Ramos, M.A. (2014). Masculinidad en el envejecimiento: vivencias de la vejez de varones de una zona popular de Lima. En J. G. Figueroa y A. Salguero ¿Y si hablas de...sde tu ser hombre? Violencia, paternidad, homoerotismo y envejecimiento en la experincia de algunos varones, 429-460. México, D.F: El Colegio de México.

Scott, A. y Wenger, C. (1996). Género y redes de apoyo social en la vejez. En S. Arber y J. Ginn: Relación entre géenro y envejecimiento, 221-240. Madrid: Narcea

UNFPA- HelpAge International (2012). Envejecimiento en el siglo XXI: una celebración y un desafio. Nueva York: Fondo de Población de Naciones Unidas (UNFPA) y HelpAge International.

Vázquez-Honorato, L.A y Salazar-Martínez, B. (2010). Arquitectura, vejez y calidad de vida. Satisfacción residencial y bienestar social. Journal of Behavior, Health \& Social Issues, 57-70. 